Bond University

Research Repository

\title{
Therapeutic ultrasound for chronic rhinosinusitis
}

Ranakusuma, Respati W.; Pitoyo, Yupitri; Safitri, Eka D.; Widyahening, Indah S.; Beller, Elaine M.; Glasziou, Paul P.; Bashiruddin, Jenny

Published in:

Cochrane Database of Systematic Reviews

DOI:

10.1002/14651858.CD011046

Licence:

Other

Link to output in Bond University research repository.

Recommended citation(APA):

Ranakusuma, R. W., Pitoyo, Y., Safitri, E. D., Widyahening, I. S., Beller, E. M., Glasziou, P. P., \& Bashiruddin, J. (2014). Therapeutic ultrasound for chronic rhinosinusitis. Cochrane Database of Systematic Reviews, 2014(3), [CD011046]. https://doi.org/10.1002/14651858.CD011046

\section{General rights}

Copyright and moral rights for the publications made accessible in the public portal are retained by the authors and/or other copyright owners and it is a condition of accessing publications that users recognise and abide by the legal requirements associated with these rights.

For more information, or if you believe that this document breaches copyright, please contact the Bond University research repository coordinator. 


\section{(E) Cochrane Library}

Cochrane Database of Systematic Reviews

\section{Therapeutic ultrasound for chronic rhinosinusitis (Protocol)}

Ranakusuma RW, Pitoyo Y, Safitri ED, Widyahening IS, Beller EM, Glasziou PP, Bashiruddin J

Ranakusuma RW, Pitoyo Y, Safitri ED, Widyahening IS, Beller EM, Glasziou PP, Bashiruddin J.

Therapeutic ultrasound for chronic rhinosinusitis.

Cochrane Database of Systematic Reviews 2014, Issue 3. Art. No.: CD011046.

DOI: 10.1002/14651858.CD011046.

www.cochranelibrary.com 
TABLE OF CONTENTS

HEADER . . . . . . . . . . . . . . . . . . . . . . . . . . . . . . . . . . . . 1

ABSTRACT . . . . . . . . . . . . . . . . . . . . . . . . . . . . . . . . . . . . . . 1

BACKGROUND . . . . . . . . . . . . . . . . . . . . . . . . . . . . . . . . . . . .

OBJECTIVES . . . . . . . . . . . . . . . . . . . . . . . . . . . . . . . . . . . . . . .

METHODS . . . . . . . . . . . . . . . . . . . . . . . . . . . . . . . . . . . . . . . .

ACKNOWLEDGEMENTS . . . . . . . . . . . . . . . . . . . . . . . . . . . . . . . . . . . . . . . .

REFERENCES . . . . . . . . . . . . . . . . . . . . . . . . . . . . . . . . . . . . . . 6

APPENDICES . . . . . . . . . . . . . . . . . . . . . . . . . . . . . . . . . . . . . . 7

CONTRIBUTIONS OF AUTHORS . . . . . . . . . . . . . . . . . . . . . . . . . . . . . . . . . . . . . . . . . . . .

DECLARATIONS OF INTEREST . . . . . . . . . . . . . . . . . . . . . . . . . . . . . . . . . . . 8

SOURCES OF SUPPORT . . . . . . . . . . . . . . . . . . . . . . . . . . . . . . . . . . . . .

Therapeutic ultrasound for chronic rhinosinusitis (Protocol)

Copyright @ 2014 The Cochrane Collaboration. Published by John Wiley \& Sons, Ltd. 


\section{[Intervention Protocol]}

\section{Therapeutic ultrasound for chronic rhinosinusitis}

Respati W Ranakusuma ${ }^{1}$, Yupitri Pitoyo ${ }^{1}$, Eka D Safitri ${ }^{1}$, Indah S Widyahening ${ }^{1}$, Elaine M Beller ${ }^{2}$, Paul P Glasziou ${ }^{3}$, Jenny Bashiruddin

${ }^{1}$ Centre for Clinical Epidemiology \& Evidence-Based Medicine, Faculty of Medicine Universitas Indonesia - Dr Cipto Mangunkusumo Hospital, Jakarta, Indonesia. ${ }^{2}$ Faculty of Health Sciences and Medicine, Bond University, Gold Coast, Australia. ${ }^{3}$ Centre for Research in Evidence-Based Practice (CREBP), Bond University, Gold Coast, Australia

Contact address: Respati W Ranakusuma, Centre for Clinical Epidemiology \& Evidence-Based Medicine, Faculty of Medicine Universitas Indonesia - Dr Cipto Mangunkusumo Hospital, 2nd Floor Building H, Jl. Diponegoro 71, Jakarta, 10430, Indonesia. anggiranakusuma@yahoo.com.

Editorial group: Cochrane ENT Group.

Publication status and date: New, published in Issue 3, 2014.

Citation: Ranakusuma RW, Pitoyo Y, Safitri ED, Widyahening IS, Beller EM, Glasziou PP, Bashiruddin J. Therapeutic ultrasound for chronic rhinosinusitis. Cochrane Database of Systematic Reviews 2014, Issue 3. Art. No.: CD011046. DOI: 10.1002/14651858.CD011046.

Copyright (C) 2014 The Cochrane Collaboration. Published by John Wiley \& Sons, Ltd.

\section{A B S T R A C T}

This is the protocol for a review and there is no abstract. The objectives are as follows:

To assess the effectiveness of ultrasound as treatment for CRS.

\section{B A C K G R O U N D}

\section{Description of the condition}

\section{Definition of chronic rhinosinusitis (CRS)}

Rhinosinusitis is defined as "inflammation of the nose and the paranasal sinuses characterised by two or more symptoms, one of which should be either nasal blockage/obstruction/congestion or nasal discharge (anterior or posterior nasal drip) with/without facial pain or pressure, reduction or loss of smell, and either endoscopic signs of nasal polyps, and/or mucopurulent discharge primarily from middle meatus and/or oedema/mucosal obstruction primarily in middle meatus and/or CT changes (mucosal changes within the ostiomeatal complex and/or sinuses)" (EPOS statement: Fokkens 2012).
When the symptoms have not completely resolved in 12 weeks or more, the disease is defined as chronic (Fokkens 2012). In some cases of CRS, nasal polyps can be found. A variety of factors contribute to the development of CRS and many studies have been conducted to find the best treatment. Several factors may predispose people to CRS, including infection, allergy and asthma, impaired mucociliary clearance, hormonal changes (e.g. pregnancy) and genetic factors (Fokkens 2012; Tan 2010). To date, there is no evidence of an aetiological correlation between anatomic variation of the paranasal sinuses and CRS (Fokkens 2012). However, a review has suggested that the underlying anatomy of sinus drainage, leading to ostial obstruction, is one exacerbating factor in CRS (Timperley 2010). Another study has found that in the majority of CRS cases, persistent inflammation could cause obstruction of the osteomeatal complex, which could explain the pathogenesis of CRS (Tan 2010). One of the barriers to effective CRS treatment is the 'bacterial biofilm', an extracellular matrix or 'film' which

Therapeutic ultrasound for chronic rhinosinusitis (Protocol)

Copyright ( 2014 The Cochrane Collaboration. Published by John Wiley \& Sons, Ltd. 
covers and protects the bacteria, and which might later lead to a recurrence of CRS (Ansari 2007; Fokkens 2012; Harvey 2007).

\section{Diagnosis}

Diagnosis of CRS requires a systematic examination starting with assessment of the symptoms and supported by physical, nasal endoscopic and radiological examination (e.g. computerised tomography (CT) scan) (Tan 2010).

\section{Prevalence}

CRS is one of the most common healthcare problems worldwide. It has a significant impact on quality of life and impairs physical and social function. Although there are few epidemiological studies of CRS and its definition varies between studies, estimates of prevalence range from $5 \%$ to $15 \%$ in the urban population (IRAB 1997). In the United States, the prevalence of CRS was found to be $2 \%$ when diagnosed by doctors and identified by ICD-9 codes (Shashy 2004); based on the National Health Interview Survey this increased to $14 \%$ to $16 \%$ (Benson 1998; Cherry 2000). A study in Korea showed the overall prevalence of CRS to be approximately 7\% and that it increases with age (Kim 2011). To date there have unfortunately been few epidemiological studies concerning the prevalence and incidence of CRS without nasal polyps (CRSsNP) and with nasal polyps (CRSwNP) (Fokkens 2012).

Due to the changing patterns of antibiotic resistance, epidemiological studies of the causal pathogens in CRS are important. One study conducted in Singapore found the predominant cultures to be Staphylococcus aureus and Haemophilus influenza (H. influenzae). Another study of 83 CRS patients in the USA found culture results for coagulase-negative staphylococci (31\%), followed by $H$. influenzae (25\%), Streptococcus pneumoniae (12\%), Moraxella catarrhalis (10\%), Pseudomonas aeruginosa (7\%), alphahaemolytic streptococci (5\%) and Staphylococcus aureus (3\%). This study concluded that sensitivity testing and culture-directed therapy are needed to support treatment decision-making in CRS (Chan 2001). Further studies are needed to assess the reasons for antibiotic treatment failure in CRS that may be due to complex interactions between other pathogens (Chin 2010).

\section{Treatment}

Nasal corticosteroids and broad-spectrum antibiotics, particularly macrolides, are commonly given as therapies for CRS (Fokkens 2012). The EPOS statement reviewed the adverse effects related to the use of nasal corticosteroids (e.g. nasal irritation, bleeding and dryness) and the long-term use of antibiotics (e.g. gastrointestinal problems, rash and reversible liver dysfunction) (Fokkens 2012). Another major problem with the long-term use of antibiotics is the potential development of resistance. Regular follow-up resistance and culture tests, usually every three months, are required to monitor this (Fokkens 2012). A Cochrane review of systemic antibiotics for CRS concluded that more trials are needed to evaluate both low-dose and long-term therapy (Piromchai 2011). Functional endoscopic sinus surgery (FESS) is a potential therapy for CRS if medical treatment is unsuccessful, however few randomised controlled trials (RCTs) have been conducted to compare the efficacy of FESS with other medical therapies. A Cochrane systematic review concluded that FESS does not provide additional benefit in decreasing the symptoms of CRS (Khalil 2006). A new surgical technique in CRS treatment is endoscopic balloon sinus ostial dilation, as one of the causes of CRS is the impairment of the sinus drainage pathway of the osteomeatal complex, caused by persistent inflammation (Rocha 2011; Tan 2010). As a persistent reduction in mucosal inflammation is an important step in the treatment of CRS, the balloon is used to compress the inflamed mucosa and dilate the ostium of the sinus. However, a Cochrane review concluded that more randomised controlled trials are needed to assess the effectiveness and complications of this technique adequately (Ahmed 2011). Some minor complications associated with these procedures have been reported. Two studies have reported that $15.4 \%$ of patients experienced minor complications related to FESS: epistaxis (nosebleeds), sinus infection, stenosis (narrowing) of the middle meatal antrostomy and intranasal synechiae (adhesions) (Fairley 1993; Ragab 2004). Functional endoscopic sinus surgery requires the skills of a specialised rhinologist in order to avoid recurrence and complications and also specific equipment that may not be available in many settings. In studies of endoscopic balloon sinus ostial dilation, no major complications were found, however a common minor complication is synechiae (Ahmed 2011).

\section{Description of the intervention}

Ultrasound is one of the treatment modalities that is proposed for CRS. It is commonly used in physiotherapy to promote tissue repair, relieve muscle and joint pain, and reduce inflammation, but it has only recently been used in the treatment of rhinosinusitis (Ansari 2007; Baker 2001; Rocha 2011).

The cheek is the most common location for ultrasound application, based on the understanding that the maxillary sinuses appear to be the most common site for rhinosinusitis (Kormos 2009). In addition, adequate ciliary function is needed in the maxillary sinuses (as well as a sufficiently low viscosity of paranasal sinus secretions) because the direction of drainage is against gravity (Leung 2008). Based on this pathophysiology, the ultrasound applicator is moved over the facial skin overlying the affected sinuses (e.g. maxillary and frontal sinuses) for short durations (Kormos 2009). In three studies of CRS treatment, therapeutic ultrasound was applied at a low intensity $\left(0.5\right.$ to $\left.1 \mathrm{~W} / \mathrm{cm}^{2}\right)$ (Ansari 2007; Naghdi 2008; Rocha 2011). We have not identified any studies that have used higher intensities. 


\section{How the intervention might work}

The ultrasound waves penetrate the tissue on the way to the target organ and have two mechanisms of action (thermal and nonthermal), which may potentially work synergistically to reduce nasal symptoms (nasal blockage and secretions) (Schortinghuis 2003). The thermal effects may work by enhancing metabolic activity and circulation within the nasal tissue. The vibration created by the non-thermal mechanism may alter the consistency of nasal secretions. The intensity of the ultrasound treatment may also influence its impact, with potential beneficial effects at low intensities but with high intensities having the potential to damage exposed cells and tissues (Ansari 2007; Baker 2001; Rocha 2011). It has also been proposed that ultrasound might disrupt the extracellular matrix bonds of bacterial biofilms, reducing their protective effect. It has been suggested that therapeutic ultrasound and antibiotics might therefore potentially act together in the treatment of CRS (Bartley 2009).

A critical review has described negative side effects caused by highintensity therapeutic ultrasound in the maxillofacial region for the treatment of soft-tissue and temporomandibular disorders, whereas low-intensity ultrasound has not been found to be harmful (Schortinghuis 2003). A randomised controlled trial of ultrasound compared to antibiotics in acute bacterial rhinosinusitis showed mild adverse effects consisting of nausea/stomach pain and headache $\left(\mathrm{H}_{\dot{\varnothing}}\right.$ Só ien 2010). Young et al, in preliminary observations (a clinical trial with a pre-test post-test study design), reported one adverse event, which was the development of acute rhinosinusitis after ultrasound therapy, but no other adverse events (Young 2010).

\section{Why it is important to do this review}

Currently, there are many treatment modalities used for the treatment of CRS, including ultrasound. There are some published clinical studies on therapeutic ultrasound used as treatment or adjuvant therapy for CRS, including randomised controlled trials (Ansari 2007; Naghdi 2008; Young 2010), but no systematic reviews. A systematic review is needed to bring to this limited evidence together in one place and to assess the effectiveness of ultrasound for the treatment of CRS.

\section{O B JECT IVES}

To assess the effectiveness of ultrasound as treatment for CRS.

\section{METHODS}

\section{Criteria for considering studies for this review}

\section{Types of studies}

Randomised controlled trials (RCTs) or quasi-randomised controlled trials.

\section{Types of participants}

We will include patients of any age (children and adults) with CRS as defined in either:

- the European Position Paper on Rhinosinusitis and Nasal Polyps 2012 (Fokkens 2012); or

- the Rhinosinusitis Task Force Report (RTFR 1996) and its revision by the Sinus and Allergy Health Partnership Task Force (including the members of RTFR 1996 from the American Academy of Otolaryngology - Head and Neck Surgery)

(Benninger 2003); or

- sinusitis symptoms with/without endoscopic evidence and/ or radiological evidence of sinusitis for 12 weeks or more (Khalil 2006); or

- no complete resolution of rhinosinusitis symptoms after 12 weeks or more (e.g. no treatment, failed medical therapy) (Fokkens 2012).

We will exclude patients in whom ultrasound is contraindicated, e.g. patients with a pacemaker, cancer, pregnancy or impaired vascular circulation (Batavia 2004).

\section{Types of interventions}

\section{Intervention}

The intervention is ultrasound given as a treatment for CRS, either continuous or pulsed, at any intensity or frequency and for any number of sessions or duration.

\section{Comparison}

The comparisons will be sham ultrasound therapy, no treatment or other treatment.

\section{Types of outcome measures}

\section{Primary outcomes}

1. Resolution of symptoms or reduction in overall symptom severity. Reduction in symptom severity may be measured using visual analogue scales or a validated symptom scale, e.g. Sinonasal Outcome Test/SNOT-20 (Piccirillo 2002).

2. Reduction in overall symptom duration.

3. Adverse effects of treatment.

Therapeutic ultrasound for chronic rhinosinusitis (Protocol)

Copyright $\odot 2014$ The Cochrane Collaboration. Published by John Wiley \& Sons, Ltd. 


\section{Secondary outcomes}

1. Reduction in other symptom scores/scales, which are reported by a majority of studies.

2. Recurrence of symptoms, defined as new episodes of rhinosinusitis or the proportion of patients with recurrent rhinosinusitis after four weeks from the start of therapeutic ultrasound.

3. Improvement in objective measures of disease (e.g. by nasal endoscopic or CT examination).

\section{Search methods for identification of studies}

We will conduct systematic searches for randomised controlled trials. There will be no language, publication year or publication status restrictions. We may contact original authors for clarification and further data if trial reports are unclear, and we will arrange translations of papers to the English language where necessary.

\section{Electronic searches}

We will identify published, unpublished and ongoing studies by searching the following databases from their inception: the Cochrane Ear, Nose and Throat Disorders Group Trials Register; the Cochrane Central Register of Controlled Trials (CENTRAL, The Cochrane Library); PubMed; EMBASE; CINAHL; LILACS; KoreaMed; IndMed; PakMediNet; CAB Abstracts; Web of Science; BIOSIS Previews and AMED. We will search the trials registries ISRCTN, ClinicalTrials.gov and ICTRP to locate any ongoing unpublished trials.

We will model search strategies for databases on the one designed for CENTRAL (Appendix 1). Where appropriate, we will combine subject strategies with adaptations of the highly sensitive search strategy designed by The Cochrane Collaboration for identifying randomised controlled trials and controlled clinical trials (as described in the Cochrane Handbook for Systematic Reviews of Interventions Version 5.1.0, Box 6.4.b (Handbook 2011).

\section{Searching other resources}

We will scan the reference lists of identified publications for additional trials and contact trial authors if necessary. We will search PubMed, TRIP database, The Cochrane Library and Google to retrieve existing systematic reviews relevant to this systematic review, so that we can scan their reference lists for additional trials. We will search for conference abstracts using the Cochrane Ear, Nose and Throat Disorders Group Trials Register.

\section{Data collection and analysis}

\section{Selection of studies}

Two authors (RR and YP) will analyse the titles and abstracts from the searches. We will acquire the full text of studies that potentially meet the eligibility criteria. We will also obtain full-text articles if eligibility of the study cannot be determined due to insufficient information supplied in the abstract or in the absence of an abstract. The same two authors will independently assess study eligibility from the full text to ensure they meet the inclusion criteria for the review. We will resolve any disagreements over which studies to include by discussion and consensus or if disagreement cannot be resolved by these methods, we will consult a third author (EB). Where clarification is required, we will contact the study authors to request the relevant information. We will translate studies reported in non-English language journals before assessment. Where more than one publication of a study exists, we will group reports together and use the publication with the most complete data in the analyses. Where relevant outcomes are only published in earlier versions, we will use these data. We will highlight any discrepancy between published versions.

We will document reasons for the exclusion of studies.

\section{Data extraction and management}

Two review authors (RR and ES) will independently extract data, using a standard data extraction form developed by the review authors for the purpose of the review. The extraction form includes the following information:

1. Sources: year of publication, citation and contact details.

2. Methods: study design, randomisation procedure, allocation, blinding (participants, people administering treatment, outcome assessors), duration of study, analysis method.

3. Participants: number, setting (primary or referral centres), diagnostic criteria of CRS, age and sex.

4. Interventions: interventions (intensity, frequency, exposure mode, duration), comparison group treatment given.

5. Outcomes: outcomes specified above, any other outcomes assessed in the trial, other events, length of follow-up.

6. Results: number of participants allocated to each intervention group and also for each outcome and time of assessment specified above, including a measure of variation.

\section{Assessment of risk of bias in included studies}

JB and ISW will undertake assessment of the risk of bias of the included trials independently, with the following taken into consideration, as guided by theCochrane Handbook for Systematic Reviews of Interventions (Handbook 2011):

1. random sequence generation (selection bias);

2. allocation concealment (selection bias);

3. blinding of participants and personnel (performance bias);

4. blinding of outcome assessment (detection bias);

5. incomplete outcome data (attrition bias);

Therapeutic ultrasound for chronic rhinosinusitis (Protocol)

Copyright @ 2014 The Cochrane Collaboration. Published by John Wiley \& Sons, Ltd. 
6. selective reporting (reporting bias);

7. other sources of bias.

We will use the Cochrane 'Risk of bias' tool in RevMan 5.2 (RevMan 2012), which involves describing each of these domains as reported in the trial and then assigning a judgement about the adequacy of each entry: 'low', 'high' or 'unclear' risk of bias. As blinding of study participants is not possible, and the study outcomes are likely to be patient-reported, we will pay particular attention to the choice of comparator. In these circumstances, sham ultrasound will introduce less bias than a no treatment control group.

\section{Measures of treatment effect}

If possible, we will summarise data in meta-analyses, which we will perform according to the intention-to-treat (ITT) principle. This means that the number of participants randomised will be used as the denominator for each outcome. There will be three comparisons made in the following way(s):

1. ultrasound versus sham ultrasound; and/or

2. ultrasound versus no treatment; and/or

3. ultrasound versus other active treatment.

If study characteristics and observed heterogeneity suggest that it is plausible to pool the comparisons of sham ultrasound and no treatment, then we will show these as subgroups with an overall estimate also calculated.

For dichotomous outcomes (proportion with resolution of symptoms, proportion with adverse effects, proportion with recurrence), we will express results as risk ratios (RR), with 95\% confidence intervals (CI). For statistically significant results we will calculate the number needed to treat to benefit (NNTB) and the number needed to treat to harm (NNTH). Where continuous scales of measurement are used to assess the effects of treatment (reduction of symptom severity and symptom duration), we will use the mean difference (MD) or the standardised mean difference (SMD) if different scales have been used.

We recognise that some studies are likely to present results as mean change scores. If so, we will present the studies with a mean change as a subgroup separately from the other studies, but will combine the subgroups into an overall estimate. If the standard deviation of the change scores is not given in a small proportion of studies, we will impute them where possible, either using the standard deviation from similar studies, or using the correlation coefficient between baseline and final measurements if sufficient information is presented in the articles (Handbook 2011).

\section{Unit of analysis issues}

We do not expect any trials in this area to utilise a cross-over or cluster-randomised design.

For studies of more than two intervention groups, where more than two of the groups are eligible for this review, we will follow the methods of the Cochrane Handbook for Systematic Reviews of Interventions. That is, in studies with more than one control group or more than one intervention group, we will combine the results of the control or intervention groups respectively (Handbook 2011).

\section{Dealing with missing data}

Where possible, we will use intention-to-treat analyses of each included study to extract data. That is, each participant will be included in the group to which they were randomised and all randomised participants will be included in the analysis. Otherwise, we will use an available case analysis. That is, each participant for whom the outcome was measured will be included in the group to which they were randomised. We will explore the effect of any missing data using sensitivity analysis (Handbook 2011).

\section{Assessment of heterogeneity}

We will assess the presence of heterogeneity in two steps. First, we will assess obvious heterogeneity at face value by comparing populations, settings, interventions and outcomes before deciding whether it is appropriate to pool studies. Second, we will assess statistical heterogeneity by means of the $\mathrm{I}^{2}$ statistic. Thresholds for the interpretation of the $\mathrm{I}^{2}$ statistic can be misleading, since the importance of inconsistency depends on several factors. We plan to use the guide to interpretation as outlined in the Cochrane Handbook for Systematic Reviews of Interventions: $0 \%$ to $40 \%$ might not be important; $30 \%$ to $60 \%$ may represent moderate heterogeneity; $50 \%$ to $90 \%$ may represent substantial heterogeneity; $75 \%$ to $100 \%$ considerable heterogeneity (Handbook 2011).

\section{Assessment of reporting biases}

If sufficient studies are included, we will use funnel plots to assess for the potential existence of small study publication bias. We will report the conflict of interest declaration of the authors where available (Handbook 2011).

\section{Data synthesis}

Where it is reasonable to assume a single pooled effect, we will use a fixed-effect model to pool data. If a single pooled effect is not plausible, due to variation in populations and interventions, or important substantial heterogeneity, we will use a random-effects model (DerSimonian and Laird method) (Handbook 2011).

We will use RevMan 5.2 for statistical analysis (RevMan 2012).

\section{Subgroup analysis and investigation of heterogeneity}

If sufficient studies are included, we plan to subgroup the studies in the following way(s):

1. children and adults; 
2. CRSsNP (CRS without nasal polyps) and CRSwNP (CRS with nasal polyps);

3. pulsed and continuous ultrasound;

4. low-frequency and high-frequency ultrasound;

5. low-intensity and high-intensity ultrasound.

If heterogeneity is discovered at the analysis stage, which suggests other subgroup analyses, we will make it clear that these were not planned at the protocol stage (Handbook 2011).

\section{Sensitivity analysis}

We plan to conduct sensitivity analysis to determine the robustness of results to studies at high risk of bias (Handbook 2011).

\section{ACK NOW LEDGEMENTS}

This review is an unfunded project and part of collaboration for capacity-building in systematic review development between the Centre for Clinical Epidemiology and Evidence-Based Medicine Faculty of Medicine, Universitas Indonesia-Cipto Mangunkusumo Hospital, Jakarta, Indonesia and the Centre for Research in Evidence-Based Practice, Bond University, Gold Coast, Australia. It is also supported by the Australasian Cochrane Centre (Steve McDonald, Sally Green and Miranda Cumpston). We would especially like to thank Tari Turner for her assistance in protocol preparation. We would also like to thank Jenny Bellorini, Samantha Faulkner and the review panel of the Cochrane Ear, Nose and Throat Disorders Group for their support and constructive feedback.

\section{R E F E R N C E S}

\section{Additional references}

\section{Ahmed 2011}

Ahmed J, Pal S, Hopkins C, Jayaraj S. Functional endoscopic balloon dilation of sinus ostia for chronic rhinosinusitis. Cochrane Database of Systematic Reviews 2011, Issue 7. [DOI: 10.1002/14651858.CD008515.pub2]

\section{Ansari 2007}

Ansari NN, Naghdi S, Farhadi M, Jalaie S. A preliminary study into the effect of low-intensity pulsed ultrasound on chronic maxillary and frontal sinusitis. Physiotherapy Theory and Practice 2007;23(4):211-8.

\section{Baker 2001}

Baker KG, Robertson VJ, Duck FA. A review of therapeutic ultrasound: biophysical effects. Journal of the American Physical Therapy Association 2001;81:1351-8.

Bartley 2009

Bartley J, Young D. Ultrasound as a treatment for chronic rhinosinusitis. Medical Hypotheses 2009;73:15-7.

Batavia 2004

Batavia M. Contraindications for superficial heat and therapeutic ultrasound: do sources agree?. Archives of Physical Medicine and Rehabilitation 2004;85:1006-12.

Benninger 2003

Benninger MS, Ferguson BJ, Hadley JA, Hamilos DL, Jacobs M, Kennedy DW, et al. Adult chronic rhinosinusitis: definitions, diagnosis, epidemiology, and pathophysiology. Otolaryngology - Head and Neck Surgery 2003;129(3): S1-S32.

Benson 1998

Benson V, Marano MA. Current estimates from the National Health Interview Survey. Data from Vital and Health Statistics, series 10: Data from the National Health Survey 1998; Vol. 199:1-428.
Chan 2001

Chan J, Hadley J. The microbiology of chronic rhinosinusitis: results of a community surveillance study. Ear, Nose, and Throat Journal 2001;80(3):143-5.

\section{Cherry 2000}

Cherry DK, Woodwell DA. National Ambulatory Medical Care Survey. Advance Data From Vital and Health Statistics 2000;328:1-32.

\section{Chin 2010}

Chin WD, Yeak CLS, Wang DY. The microbiology and the efficacy of antibiotic-based medical treatment of chronic rhinosinusitis in Singapore. Rhinology 2010;48:433-7.

\section{Fairley 1993}

Fairley JW. Chapter 9: A prospective randomized controlled trial of functional endoscopic sinus surgery: endoscopic middle meatal antrostomy versus conventional inferior meatal antrostomy. In: Correlation of Nasal symptoms with Objective Findings and Surgical Outcome Measurement [Thesis submitted for the degree of Master of Surgery]. London, UK: University of London, 1993.

Fokkens 2012

Fokkens WJ, Lund VJ, Mullol J, Bachert C, Alobid I, Baroody F, et al. European Position Paper on Rhinosinusitis and Nasal Polyps 2012. Rhinology. Supplement 2012;23: $1-298$.

Handbook 2011

Higgins JPT, Green S (editors). Cochrane Handbook for Systematic Reviews of Interventions Version 5.1.0 [updated March 2011]. The Cochrane Collaboration, 2011. Available from www.cochrane-handbook.org.

\section{Harvey 2007}

Harvey RJ, Lund VJ. Biofilms and chronic rhinosinusitis: systematic review of evidence, current concepts and directions for research. Rhinology 2007;45:3-13. 
Hǿ Só ien 2010

$\mathrm{H}_{\varnothing}$ sø் ien E, Lund AB, Vasseljen O. Similar effect of therapeutic ultrasound and antibiotics for acute bacterial rhinosinusitis: a randomised trial. Journal of Physiotherapy 2010;56:27-32.

\section{IRAB 1997}

International Rhinosinusitis Advisory Board. Infectious rhinosinusitis in adults: classification, etiology and management. Ear, Nose, and Throat Journal 1997;76(12 Suppl):5-17.

\section{Khalil 2006}

Khalil H, Nunez DA. Functional endoscopic sinus surgery for chronic rhinosinusitis. Cochrane Database of Systematic Reviews 2006, Issue 3. [DOI: 10.1002/ 14651858.CD004458.pub2]

\section{$\operatorname{Kim} 2011$}

Kim YS, Kim NH, Seong SY, Kim KR. Prevalence and risk factors of chronic rhinosinusitis in Korea. American Journal of Rhinology \& Allergy 2011;25(3):117-21.

\section{Kormos 2009}

Kormos WA. Primary Care Medicine: Office Evaluation and Management of the Adult Patient. 6th Edition. Philadelphia: Lippincott Williams \& Wilkins, 2009.

\section{Leung 2008}

Leung RS, Katial R. The diagnosis and management of acute and chronic sinusitis. Primary Care: Clinics in Office Practice 2008;35:11-24.

\section{Naghdi 2008}

Naghdi S, Ansari NN, Farhadi M. A clinical trial on the treatment of chronic rhinosinusitis with continuous ultrasound. Journal of Physical Therapy Science 2008;20(4): 233-8.

\section{Piccirillo 2002}

Piccirillo JF, Merritt MG, Richards ML. Psychometric and clinimetric validity of the 20-Item Sino-Nasal Outcome Test (SNOT-20). Otolaryngology - Head and Neck Surgery 2002;126(1):41-7.

Piromchai 2011

Piromchai P, Thanaviratananich S, Laopaiboon M. Systemic antibiotics for chronic rhinosinusitis without nasal polyps in adults. Cochrane Database of Systematic Reviews 2011, Issue 5. [DOI: 10.1002/14651858.CD008233.pub2]

\section{Ragab 2004}

Ragab SM, Lund VJ, Scadding G. Evaluation of the medical and surgical treatment of chronic rhinosinusitis: a prospective, randomised, controlled trial. Laryngoscope 2004;114:923-30.

RevMan 2012 [Computer program]

The Nordic Cochrane Centre, The Cochrane Collaboration. Review Manager (RevMan). Version 5.2. Copenhagen: The Nordic Cochrane Centre, The Cochrane Collaboration, 2012.

\section{Rocha 2011}

Rocha WA, Rodrigues KMG, Pereira RRR, Nogueira $\mathrm{BV}$, Goncalves WL. Acute effects of therapeutic 1-MHz ultrasound on nasal unblocking of subjects with chronic rhinosinusitis. Brazilian Journal of Otorhinolaryngology 2011;77(1):7-12.

\section{RTFR 1996}

Report of the Rhinosinusitis Task Force Committee Meeting. Alexandria, Virginia, August 17, 1996. Otolaryngology - Head and Neck Surgery 1997; Vol. 117, issue $3 \mathrm{Pt} 2: \mathrm{S} 1-68$.

\section{Schortinghuis 2003}

Schortinghuis J, Stegenga B, Raghoebar GM, de Bont LG. Ultrasound stimulation of maxillofacial bone healing. Critical Reviews in Oral Biology \& Medicine 2003;14(1): 63-74.

\section{Shashy 2004}

Shashy RG, Moore EJ, Weaver A. Prevalence of the chronic sinusitis diagnosis in Olmsted County, Minnesota. Archives of Otolaryngology -- Head and Neck Surgery 2004;130(3): 320-3.

\section{Tan 2010}

Tan BK, Schleimer RP, Kern RC. Perspectives on the etiology of chronic rhinosinusitis. Current Opinion in Otolaryngology \& Head and Neck Surgery 2010;18(1):21-6.

Timperley 2010

Timperley D, Schlosser RJ, Harvey RJ. Chronic rhinosinusitis: an education and treatment model. Otolaryngology - Head and Neck Surgery 2010;143(5 Suppl 3):S3-8.

\section{Young 2010}

Young D, Morton R, Bartley J. Therapeutic ultrasound as treatment for chronic rhinosinusitis: preliminary observation. Journal of Laryngology and Otology 2010;124: 495-9.

* Indicates the major publication for the study 


\section{A P P E N D I C E S}

\section{Appendix I. CENTRAL search strategy}

\#1 MeSH descriptor: [Sinusitis] explode all trees

\#2 rhinosinusitis or nasosinusitis or sinusitis or pansinusitis or ethmoiditis or sphenoiditis

\#3 kartagener* and syndrome*

\#4 inflamm* and sinus*

$\# 5 \# 1$ or $\# 2$ or \#3 or \#4

\#6 MeSH descriptor: [Chronic Disease] explode all trees

$\# 7$ chronic or persis*

\#8 \#6 or \#7

$\# 9$ \#5 and \#8

\#10 MeSH descriptor: [Diathermy] explode all trees

\#11 ultrasound* or ultrasonic* or diatherm*

\#12 MeSH descriptor: [Ultrasonics] explode all trees

\#13 MeSH descriptor: [Radio Waves] explode all trees

\#14 MeSH descriptor: [Sound] explode all trees

\#15 "cus" or "pus" or "liust" or 1-MHz or atomiz* or atomis* or "lfu" or "swd" or "cswd" or "pswd" or "lora"

\#16 "nonthermal effect*" or "nonthermal exposure" or "thermal effect*" or "thermal exposure" or "wave exposure" or "short wave*" or "radio wave*" or shortwave*

\#17 (pulsed or continuous) and (wave* or therapy)

\#18 (acoustic* or "sound" or "high frequency" or "low frequency" or "low intensity" or elastic or sonic) and (energy or wave* or radiation)

\#19 MeSH descriptor: [Physical Therapy Specialty] explode all trees

\#20 Physiotherap* or "physical therap*"

$\# 21 \# 10$ or \#11 or \#12 or \#13 or \#14 or \#15 or \#16 or \#17 or \#18 or \#19 or \#20

$\# 22 \# 9$ and \#21

\section{CONTRIBUTIONSOFAUTHORS}

- RR will contribute as primary author and in protocol preparation, study selection and data extraction.

- ES will contribute to strategy development and study selection.

- YP will contribute to search strategy development, study selection, data extraction and data entry into RevMan.

- ISW will contribute to search strategy development, 'Risk of bias' assessment and data analysis.

- JB will contribute to 'Risk of bias' assessment and data analysis.

- EB will contribute to protocol preparation and analysis interpretation, and also with expert opinion and independent assessment.

- PG will contribute to analysis interpretation, and also with expert opinion and independent assessment.

Therapeutic ultrasound for chronic rhinosinusitis (Protocol) 


\section{DECLARATIONSOF INTEREST}

The authors have no financial or potential conflict of interest in this project.

\section{SOURCES OF SUPPORT}

\section{Internal sources}

- None, Other.

\section{External sources}

- None, Other. 